\title{
Enfermedad fúngica invasora en pacientes de cinco hospitales de la Región de Valparaíso, Chile. 2004 a 2009
}

\author{
Rodrigo Cruz Ch. y Eduardo Piontelli L.
}

\section{Invasive fungal disease in patients from five hospitals in the Valparaiso Region, Chile. 2004 to 2009}

Introduction: Invasive fungal disease (IFD) is a frequent cause of morbidity and mortality in immunocompromised patients. These infections are caused mainly by Candida albicans and Aspergillus fumigatus. Objectives: To describe IFD diagnosed by culture in adults and children from five hospitals in Valparaiso, Chile and to determine the species involved and the in vitro sensitivity of Candida spp. Material and Methods: Biodemographical data, results of cultures, antifungal treatment and mortality after 30 days, was collected. The fungi were identified by standard methods and the sensitivity of the yeasts was obtained according to the M44-A CLSI document. Results: Fifty one IFD were diagnosed, 13 in patients with haematological malignancies and 10 in immunocompetent patients from the adult ICU. The following fungal species were isolated: 34 yeasts, 15 filamentous fungi and 2 Histoplasma capsulatum. There were 33 proven and 1 probable IFD by yeasts. By filamentous fungi, there were 6 proven and 9 probable IFD, 13 out of 15 isolated species were Aspergillus (A. fumigatus being the most frequent). C. albicans was the most frequent isolated yeast, followed by $C$. tropicalis and C. parapsilosis. All were sensitive to fluconazol and voriconazol, excepting $C$. glabrata. The mortality by Candida and by filamentous fungi was $42.42 \%$ and $86.66 \%$ respectively. Conclusions: IFD was diagnosed mainly in immunocompromised and adult ICU patients. The mortality rate in IFD by filamentous fungi was higher than Candida group. The main agents involved were Candida albicans and Aspergillus fumigatus. A high sensitivity to fluconazol and voriconazol was found in strains of Candida.

Key words: Invasive fungal disease, immunocompromise, Aspergillus, Candida, Mortality.

Palabras clave: Enfermedad fúngica invasora, inmunocompromiso, Aspergillus, Candida, mortalidad.

\section{Introducción}

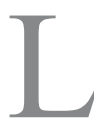

a enfermedad fúngica invasora (EFI) es una causa frecuente de morbimortalidad en pacientes inmunocomprometidos ${ }^{1-3}$. Estas infecciones son más frecuentes en los últimos años debido al aumento de los trasplantes, tratamientos más inmunodepresores, citotóxicos y mayor número de pacientes con $\operatorname{SIDA}^{4,5}$.

Las EFI son provocadas, en más de $80 \%$, por levaduras del género Candida y hongos filamentosos pertenecientes al genero Aspergillus. Si bien Aspergillus fumigatus (Eurotiomycetes) predomina ampliamente en las EFI provocadas por hongos filamentosos, otras especies de Aspergillus (A. flavus, A. niger, A. terreus y A. nidulans), Fusarium spp (Hypocreales), Scedosporium spp (Microascaceae), Paecilomyces spp (SordariomycetidaeEurotiomycetidae) y taxas pertenecientes a los Mucormycotina, han incrementado su incidencia a partir de los años noventa, especialmente en pacientes sometidos a trasplantes de progenitores hematopoyéticos, rechazo de trasplante y en pacientes neutropénicos ${ }^{6-8}$. Este cambio de tendencia puede relacionarse no sólo con un aumento real de las micosis invasoras, sino también a una mayor sospecha de estas entidades, mejores pruebas diagnósticas -marcadores serológicos de antígeno de galactomanano y $1 \rightarrow 3$ - $\beta$-D-glucano, avances en el uso e interpretación de los estudios de imágenes-, uso de antimicóticos profilácticos y cambios en los procedimientos de trasplantes ${ }^{9,10-13}$.

En el caso de las EFI por levaduras, Candida albicans sigue siendo el principal agente; sin embargo, han aumentado otras especies de Candida, Trichosporon spp, Rhodotorula spp y Saccharomyces $\mathrm{spp}^{7,10,14}$.

La importancia relativa de las EFI y sus diferentes agentes puede variar en las distintas zonas geográficas, por lo que es de gran utilidad conocer la epidemiología local y regional en los centros asistenciales de los diferentes países ${ }^{15,16}$.

El objetivo de este trabajo fue describir las EFI diagnosticadas con cultivo en pacientes adultos y niños, con y sin inmunocompromiso, de cinco hospitales de la Región

\author{
Universidad de \\ Valparaíso, Chile. \\ Facultad de Medicina \\ Cátedra de Micología (RCCH, EPL). \\ Hospital San Camilo, \\ San Felipe, Región de \\ Valparaíso, Chile \\ Unidad de Cuidados Intensivos \\ (RCCH). \\ Hospital Carlos Van Buren, \\ Valparaíso, Chile \\ Infecciones Intrahospitalarias \\ ( $\mathrm{RCHH}$ ). \\ Los autores declaran no tener \\ conflictos de interés. \\ Financiamiento: fondos propios. \\ Recibido: 7 de julio de 2010 \\ Aceptado: 6 de enero de 2011
}

Correspondencia a: Rodrigo Cruz Choappa rcruzchoappa@gmail.com 
de Valparaíso, además de conocer la frecuencia de las especies involucradas y, en el caso de los integrantes del genero Candida, determinar la relación albicans /no albicans y la sensibilidad in vitro a fluconazol y voriconazol.

\section{Material y Método}

Estudio descriptivo, prospectivo. Se seleccionaron todos aquellos pacientes adultos y niños, con o sin inmunocompromiso, en quienes se identificó un episodio de EFI diagnosticado con cultivo, durante el período comprendido entre enero de 2004 y diciembre de 2009, en cinco hospitales de la región (H. Carlos van Buren de Valparaíso, H. Naval y H. Gustavo Fricke de Viña del Mar, H. San Camilo de San Felipe y H. Dr. Eduardo Pereira R. de Valparaíso). Los datos fueron obtenidos de las fichas clínicas de los pacientes e información aportada por los médicos tratantes.

\begin{tabular}{|lccc|}
\hline \multicolumn{3}{|c|}{ Tabla 1. Características generales de los } \\
pacientes con EFI
\end{tabular}

Tabla 2. Factores de riesgo conocidos para EFI

Por levaduras

\begin{tabular}{lclc} 
Factor de riesgo & n & Factor de riesgo & n \\
AM amplio espectro & 27 & Neutropenia & 14 \\
CVC & 23 & Infección por VIH/SIDA & 4 \\
Cirugía reciente & 8 & Cetoacidosis diabética & 1 \\
NTP & 4 & Fibrosis quística & 1 \\
Terapia inmunosupresora & 4 & Peritoneodialisis & 1 \\
\hline AM: antimicrobiano CVC. catéter venoso central. NTP. nutrición parenteral total. &
\end{tabular}

El diagnóstico de EFI fue realizado por el equipo tratante frente a cada caso particular, considerando la condición clínica de los pacientes, las imágenes (radiografías, tomografía axial computada, resonancia magnética) y cultivos micológicos, además de aplicar los criterios EORTC/MSG en los casos que correspondiera ${ }^{17}$.

En los casos identificados se completó una ficha que incluyó antecedentes demográficos, enfermedad de base, factores de riesgo conocidos en la literatura médica para $\mathrm{EFI}^{4,18,19}$, resultados de cultivos, tratamiento antifúngico y mortalidad a 30 días.

La identificación final de cada especie se realizó en la Cátedra de Micología de la Universidad de Valparaíso. La identificación de hongos filamentosos se realizó por morfología y pruebas fisiológicas. En el caso de las levaduras se utilizó morfología, pruebas fisiológicas y bioquímicas $^{20-22}$. La sensibilidad a fluconazol y voriconazol de las levaduras fue determinada por método de difusión en discos según el documento M44-A del CLSI ${ }^{23-25}$.

En el análisis estadístico se usó el programa Microsoft Excel 2003, los resultados de las variables nominales se expresaron en porcentajes y de las numéricas en promedio.

\section{Resultados}

Durante el período estudiado se identificaron 51 episodios de EFI, 13 en pacientes onco-hematológicos, 10 en pacientes inmunocompetentes en UCI adultos, 7 casos en pacientes con infección por VIH/SIDA (todos con recuento de CD4 menor de 100 céls $/ \mathrm{mm}^{3}$ ) y 21 casos con otras características generales (Tabla 1).

El promedio de edad de todos los paciente fue de 36,3 años (20 días - 77 años), de los cuales 50 son chilenos y uno colombiano, 23 (45\%) mujeres y 39 sobre 15 años de edad.

Según criterios EORTC/MSG hubo 33 infecciones probadas por levaduras (32 casos por Candida spp y un caso por Cryptococcus neoformans) y una probable (paciente neutropénico, con exudados en la retina y cultivo en orina para C. tropicalis).

En el caso de las EFI por hongos filamentosos se diagnosticaron seis probadas y nueve probables (todos neutropénicos, con compromiso pulmonar al TAC y cultivos para Aspergillus del LBA). Además se describieron dos casos de histoplasmosis diseminada demostrada.

Los factores de riesgo descritos en la literatura científica que presentaron los pacientes con EFI por cándida fueron principalmente: uso previo de antimicrobianos de amplio espectro y la instalación de catéter venoso central (CVC), mientras que en los pacientes con EFI por hongos filamentosos fue neutropenia, independiente de la causa que la produjo (Tabla 2). Ninguno de los pacientes recibió tratamiento antifúngico profiláctico.

De los 33 casos de candidiasis, 26 fueron tratados con 
Experiencia Clínica

fluconazol, tres con anfotericina B deoxicolato, dos con caspofungina y dos casos recibieron secuencialmente fluconazol y caspofungina. Hubo un caso de meningitis por $C$. neoformans en un paciente sometido a trasplante renal, que fue tratado con anfotericina $\mathrm{B}$ deoxicolato. En cambio, de los 15 casos de EFI por hongos filamentosos, 11 fueron tratados con anfotericina $\mathrm{B}$ deoxicolato, dos con voriconazol, un caso recibió secuencialmente anfotericina B y voriconazol, y otro anfotericina B, voriconazol e itraconazol. Los dos casos de histoplasmosis fueron tratados con anfotericina $\mathrm{B}$ deoxicolato.

La mortalidad global en EFI por especies de Candida fue de 42,4\% (14/33); sin embargo, en las EFI por hongos filamentosos se elevó hasta 86,6\%(13/15). Los dos casos de histoplasmosis tuvieron resultado fatal.

En total se aislaron 51 especies fúngicas, de las cuales $34(66,6 \%)$ correspondieron a levaduras, $15(29,4 \%)$ a hongos filamentosos y dos casos a Histoplasma capsulatum. Las levaduras se aislaron principalmente de sangre y los hongos filamentosos de cultivos de lavado broncoalveolar (Tabla 3).

Entre las levaduras, la especie con mayor frecuencia fue $C$. albicans, seguida de C. tropicalis y C. parapsilosis, mientras que $C$. glabrata se aisló solo en dos ocasiones (Tabla 3), además se observó que las especies no albicans en conjunto $(57,5 \%)$ fueron más frecuentes que $C$. albicans $(42,2 \%)$.

Con respecto a la sensibilidad de las especies del género Candida, todas fueron sensibles a fluconazol y a voriconazol, excepto $C$. glabrata, que fue resistente en una de las dos cepas aisladas. La cepa de C. neoformans aislada en LCR también fue sensible a los dos antifúngicos testeados.

En el caso de las EFI por hongos filamentosos, $13 \mathrm{de}$ 15 especies aisladas $(86,66 \%)$ correspondieron al genero Aspergillus, siendo A. fumigatus el más frecuente $8 / 13$ $(61,5 \%)$. Hubo un caso de peritonitis por Trichoderma longibrachiatum en una niña de 13 años en peritoneo-diálisis y se destaca la presencia de dos casos de histoplasmosis diseminada en pacientes infectados por VIH, en etapa SIDA, provenientes de zonas endémicas (Colombia y Norte de Argentina, respectivamente) para esta micosis (Figura 1).

Figura 1. Microscopia DIC. Aumento 1000X: a. Aspergillus fumigatus, conidios y conidióforos; b. Aspergillus flavus, vesícula biseriada, conidios y conidióforo rugoso incoloro; c. Emericela nidulans, vesícula biseriada, conidios y conidióforo café; $\mathbf{d}$. Aspergillus niger, métulas y fiálides con conidios rugosos separados de su vesícula; e. Trichoderma logibrachiatum, disposición triangular de las ramas del conidióforo, fiálides y conidios elipsoidales; f. Histoplasma capsulatum, macroconidios rugosos (digitiformes) y algunos microconidios.

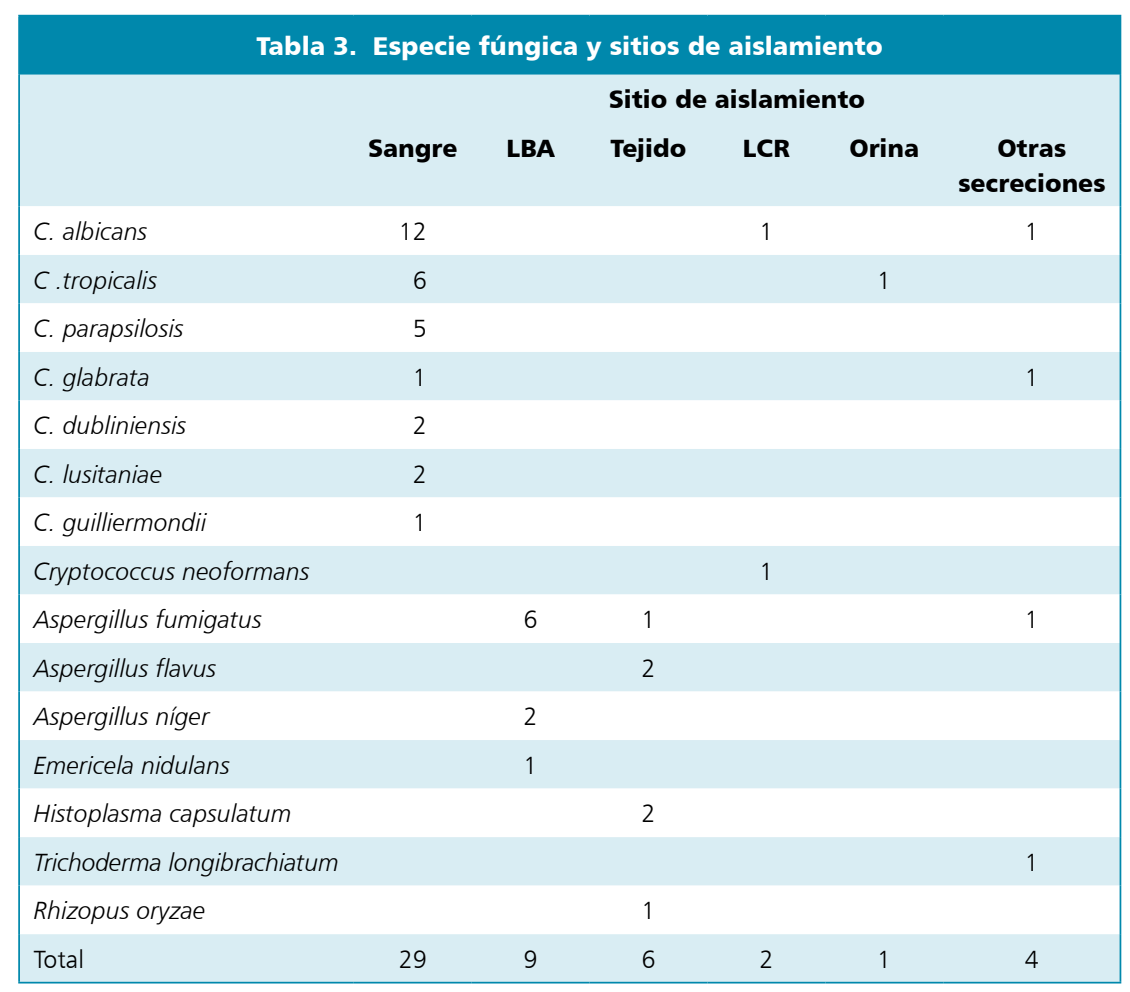

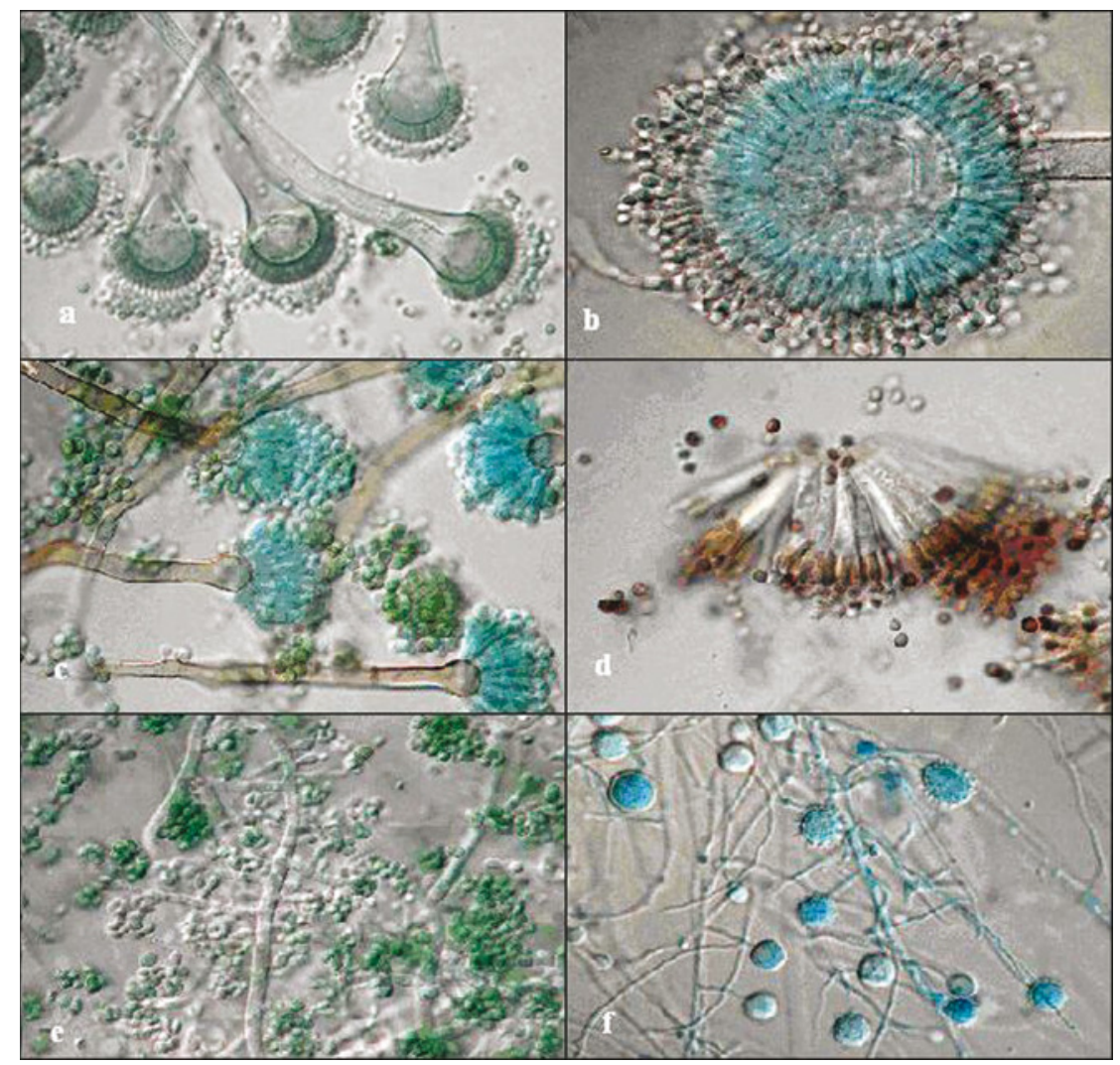




\section{Discusión}

Los casos de EFI diagnosticados en la Región de Valparaíso durante el período señalado se caracterizaron por su heterogeneidad, tanto por los grupos etáreos, como por las múltiples patologías de base y factores de riesgo presentados.

La mayoría de los episodios de EFI ocurrió en pacientes onco-hematológicos, especialmente las provocadas por hongos filamentosos; sin embargo, se presentaron casos en otros grupos de pacientes, como SIDA y enfermedad granulomatosa crónica. La experiencia nacional e internacional es amplia en EFI por hongos filamentosos en pacientes onco-hematológicos, tanto en adultos como en niños, siendo los pacientes con leucemia mieloide aguda (LMA) los que presentan el mayor número de episodios de estas infecciones; no obstante, la descripción de casos, series y revisiones en otros grupos de pacientes con otros factores de riesgo se encuentra ampliamente documentada ${ }^{9,15,26-30}$. En nuestra serie, la mayoría de los pacientes onco-hematológicos tenía LMA y debido a que en nuestra región no se realizan trasplante de progenitores hematopoyéticos (otro grupo de pacientes con riesgo para infecciones invasoras por hongos), no se describieron estos casos.

Las EFI por levaduras afectaron principalmente a pacientes inmunocompetentes de UCI con CVC y tratamiento antimicrobiano de amplio espectro, además de pacientes con cirugía abdómino-pélvica reciente. En menor medida se presentaron en pacientes inmunodeprimidos, a diferencia de lo que ocurrió con las EFI por hongos filamentosos, situaciones que concuerdan, tanto con lo publicado a nivel nacional como internacional ${ }^{9,31-33}$. Se destaca un caso fatal de meningitis por C. neoformans en un paciente con trasplante renal en tratamiento inmunosupresor.

En el caso de los pacientes con SIDA, todos tenían recuento de CD4 menor a 100 céls $/ \mathrm{mm}^{3}$ y cuatro tenían, además, neutropenia asociada. Ellos presentaron, tanto infecciones por levaduras como por hongos filamentosos y, en dos casos, infecciones diseminadas alóctonas (Colombia y norte de Argentina) por H. capsulatum, ambos con resultado fatal. Desde la aparición del VIH, se han descrito en todo el mundo, tanto infecciones superficiales como profundas en estos pacientes, destacando la neumocistosis, candidiasis, criptococosis y aspergilosis, además de infecciones por hongos dimórficos en aquellas zonas endémicas ${ }^{34-36}$.

La mayoría de los diagnósticos de EFI por levaduras fue con aislamiento del hongo en sangre, por lo que correspondieron a diagnósticos de certeza; sin embargo, en el caso de las EFI por hongos filamentosos predominaron los cultivos a partir de LBA, por lo que muchos diagnósticos se debieron de catalogar de probables, según los criterios de EORTC/MSG $(9,17)$.En nuestra serie no se utilizaron marcadores serológicos como $1 \rightarrow 3-\beta$-D-glucano o galactomanano en el diagnóstico de las EFI, exámenes que en la actualidad son una herramienta útil y precoz en el diagnóstico de estas infecciones ${ }^{1,4,9,15,17,37,38}$.

La mortalidad global a 30 días en EFI por hongos filamentosos y por levaduras fue más elevada de lo que reportan los últimos trabajos, lo que puede deberse al retraso en el diagnóstico, co-morbilidad que presentaron los pacientes y a que en el caso de aspergilosis el principal tratamiento utilizado fue anfotericina $\mathrm{B}$ deoxicolato, el cual ha demostrado tener menor efectividad y mayores efectos adversos que medicamentos como voriconazol y caspofungina $^{39,40}$. Lass-Flörl ${ }^{41}$, describe en Europa una mortalidad de aspergilosis invasora de $38 \%$ en paciente con leucemia aguda y hasta $94 \%$ en pacientes no neutropénicos. Para candidiasis describe una mortalidad que fluctuó entre 28 y 59\% dependiendo de la especie y la localización de la infección. En el estudio ECMM, Tortorano y cols ${ }^{42}$, encontraron una mortalidad para candidemia de $37 \%$ a 30 días, la que fue superior en los subgrupos de ancianos (49\%), pacientes oncológicos (49\%), hematológicos $(45 \%)$ y críticos $(42,4 \%)$. Upton y cols ${ }^{43}$, en E.U.A. reportaron una mortalidad atribuible a 30 días para aspergilosis invasora de $33 \%$ en pacientes con trasplante de progenitores hematopoyéticos. En Chile, Rabagliati y cols ${ }^{15}$, reportaron $33 \%$ de mortalidad en los casos de aspergilosis y $14 \%$ en candidiasis en pacientes onco-hematológicos.

Fluconazol fue el medicamento más utilizado en candidiasis y como la principal especie fue C. albicans, la cual era sensible a fluconazol, la decisión terapéutica en general no fue complicada, a diferencia de la realidad de otros lugares donde se recomienda inicio de tratamiento con caspofungina o anfotericina $\mathrm{B}$ liposomal debido a que las especies no albicans resistentes al fluconazol han ido en franco aumento ${ }^{44,45}$.

Las EFI por levaduras fueron las más frecuentes, predominando $C$. albicans; no obstante, en conjunto las especies no albicans predominaron (sobre $50 \%$ del total), siendo C. tropicalis y C. parapsilosis las más frecuentes. Reboli y cols ${ }^{19}$, en E.U.A. reportaron 59 a $64 \%$ de los casos por C. albicans seguido por C. glabrata con 16 a $25 \%$. St-Germain y cols ${ }^{46}$, en Canadá reportaron $54 \%$ de C. albicans seguido por C. glabrata con una frecuencia de $15 \%$ y $C$. parapsilosis con $12 \%$, situaciones distintas a nuestra realidad, donde $C$. glabrata se encuentra escasamente, lo que es de suma importancia ya que ésta se asocia con una mayor resistencia a los azoles. Gómez y cols ${ }^{47}$, en España describieron los cambios entre los periodos 1993-1998 y 2002-2005, donde C. albicans disminuyó desde $66,2 \%$ hasta $45,8 \%$ y las especies no-albicans aumentaron desde $33,7 \%$ hasta $54,2 \%$. En Chile, Silva y cols $^{14}$, en el 2002 reportaron $43 \%$ de C. albicans seguido por C. parapsilosis con $19 \%$, C. tropicalis con $13 \%$ y $C$. 
glabrata sólo con 3,3\%, datos que se asemejan con los resultados obtenidos en este trabajo.

Con respecto a la sensibilidad, todas las cepas, excepto una de C. glabrata, fueron sensibles in vitro a fluconazol y a voriconazol, situación favorable ya que fluconazol es el principal antifúngico en el tratamiento de las candidiasis en la mayoría de los países en desarrollo, debido a su menor costo.

En el trabajo de Silva y cols ${ }^{14}$, no encontraron resistencia de C. albicans a fluconazol; sin embargo, $17 \%$ de las cepas de $C$. tropicalis y $5 \%$ de $C$. parapsilosis fueron resistentes a este antifúngico, situación distinta a nuestra experiencia donde no hubo resistencia a fluconazol en estas especies. No obstante, se debe precisar que la metodología para determinar la sensibilidad fue por método de difusión en discos según el documento M44-A del CLSI; en cambio, en el trabajo de Silva y cols. se utilizó el método estándar de macro y microdilución según documento M27-A del NCCLS. Además, la procedencia de las cepas fue distinta, las nuestras procedieron exclusivamente de la Región de Valparaíso y las del trabajo en comparación, de varios centros asistenciales de Santiago $\mathrm{y}$ de otras regiones.

En EFI por hongos filamentosos predominó el género Aspergillus y dentro de éste A. fumigatus; sin embargo, aparecieron otras especies como A. flavus, A. niger y $A$. nidulans, que en los últimos años han aumentado en los aislamientos, principalmente en pacientes oncohematológicos $5,15,39$.

Cabe destacar el primer diagnóstico en Chile de peritonitis por T. logibrachiatum en una niña de 13 años, quien se encontraba en peritoneo-diálisis, agente que se describe esporádicamente en la literatura médica provocando infecciones invasoras en distintos órganos ${ }^{48,49}$ y un caso de zigomicosis rinocerebral por Rhizopus oryzae en una paciente con cetoacidosis diabética. La forma rinosinusal que presentó nuestra paciente generalmente se asocia con diabetes mellitus; se describen otras localizaciones como pulmonar, cutáneo-articular, renal y diseminada ${ }^{50-52}$.

Sin duda, nuestra serie refleja una realidad parcial de lo que sucede con las micosis invasoras en nuestra región, debido a que se incluyeron sólo las EFI diagnosticadas con cultivos positivos; aún así, es un avance en conocer la situación local, lo cual es la base para poder planificar la prevención, el diagnóstico y el tratamiento, enfrentando así de mejor forma estas infecciones con los recursos muchas veces limitados de las instituciones públicas.
Agradecimientos. Por su colaboración y sus generosos aportes a Rodrigo Vergara y Gonzalo Wilson. Hospital Carlos Van Buren, Isabel Briceño y Elizabeth Barthel. Hospital Naval, Paulina Díaz y Marcela González. Hospital Gustavo Fricke, UCI Hospital San Camilo, San Felipe y a los profesionales de Microbiología en los hospitales Carlos van Buren, Dr. Eduardo Pereira R. y Gustavo Fricke. También agradecemos por sus sugerencias y correcciones del texto a Patricia Vásquez. Hospital San Juan de Dios, Luis Thompson. Clínica Alemana y a M. Cristina Díaz, Micología, Instituto de Ciencias Biomédicas, Universidad de Chile.

\section{Resumen}

Introducción: La enfermedad fúngica invasora (EFI) es una causa frecuente de morbimortalidad en pacientes inmunocomprometidos. Es provocada principalmente por Candida albicans y Aspergillus fumigatus. Objetivos: Describir las EFI diagnosticadas con cultivos en adultos y niños de cinco hospitales de la Región de Valparaíso, Chile, conocer las especies involucradas y para Candida spp determinar su sensibilidad in vitro. Material y Métodos: En cada caso se recopilaron antecedentes demográficos, cultivos, tratamiento antifúngico y mortalidad a 30 días. La identificación de hongos se realizó por métodos estandarizados y la sensibilidad de levaduras según documento M44-A del CLSI. Resultados: se diagnosticaron $51 \mathrm{EFI}$, destacando 13 en pacientes onco-hematológicos y 10 en inmunocompetentes de UCI adultos. Se aislaron 34 levaduras, 15 hongos filamentosos y en dos casos Histoplasma capsulatum. Hubo 33 EFI probabas por levaduras y una probable. En EFI por hongos filamentosos 6 fueron probadas y 9 probables; 13 de 15 especies aisladas fueron Aspergillus, siendo $A$. fumigatus la más frecuente. Entre las levaduras predominó C. albicans, seguida de C. tropicalis y C.parapsilosis. Todas fueron sensibles a fluconazol y voriconazol, excepto C. glabrata. La mortalidad de EFI por Candida fue de $42,4 \%$ y de EFI por hongos filamentosos de $86,6 \%$. Conclusiones: la EFI se diagnosticó principalmente en pacientes inmunocomprometidos y en pacientes de UCI adultos. La mortalidad de EFI por hongos filamentosos fue mayor que la causada por Candida. Los principales agentes fueron $C$. albicans y A. fumigatus. Existe una alta sensibilidad de las cepas de Candida spp a fluconazol y voriconazol.

\section{Referencias}

1.- Ascioglu S, Rex J H, De Pauw B, Bennett J E, Bille J, Crokaert F, et al. Defining opportunistic invasive fungal infections in immunocompromised patients with cancer and hematopoietic stem cell transplants: An International Consensus. Clin Infect Dis 2002; 34: 7-14.

2.- Chamilos G, Luna M, Lewis R E, Bodey G $\mathrm{P}$, Chemaly R, Tarrand J J, et al. Invasive fungal infections in patients with hematologic malignancies in a tertiary care cancer center: an autopsy study over a 15 year period (19892003). Hematological 2006; 91: 986-9.

3.- Horn D L, Neofytos D, Anaissie E J, Fishman J A, Steinbach W J, Olyaei A J, et al. Epidemiology and outcomes of candidemia 
in 2019 patients: data from the prospective antifungal therapy alliance registry. Clin Infect Dis 2009; 48: 1695-703.

4.- García-Ruiz JC, Amutio E, Ponton J. Infección fúngica invasora en pacientes inmunodeficientes. Rev Iberoam Micol 2004; 21: 55-62

5.- Enoch D A, Ludlam H A, Brown N M. Invasive fungal infections: a review of epidemiology and management options. J Med Microbiol 2006; 55: 809-18.

6.- Marr K A, Carter R A, Crippa F, Wald A, Corey L. Epidemiology and outcome of mould infections in hematopoietic stem cell transplant recipients. Clin Infect Dis 2002; 34: 909-17.

7.- Marr K A, Crippa F, Leisenring W, Hoyle M, Boeckh M, Balajee S A, et al. Itraconazole versus fluconazole for prevention of fungal infections in patients receiving allogeneic stem cell transplants. Blood 2004; 103: 1527-33.

8.- Husain S, Alexander B D, Munoz P, Avery R K, Houston S, Pruett T, et al. Opportunistic mycelial fungal infections in organ transplant recipients: emerging importance of nonAspergillus mycelial fungi. Clin Infect Dis 2003; 37: 221-9.

9.- Pontón J, Quindós G, editores. Micosis invasoras en los pacientes oncohematológicos. Guía de bolsillo. Sociedad española de Micología. 2009.

10.- Pagano L, Caira M, Nosari A, Van Lint M T, Candoni A, Offidani M, et al. Fungal infections in recipients of hematopoietic stem cell transplants: results of the SEIFEM B-2004 study-Sorveglianza Epidemiológica Infezioni Fungine Nelle Emopatie Maligne. Clin Infect Dis 2007; 45: 1161-70.

11.- Maertens J, Verhaegen J, Lagrou K, Van Eldere J, Boogaerts M. Screening for circulating galactomannan as a noninvasive diagnostic tool for invasive aspergillosis in prolongued neutropenic patients and stem cell transplantation recipients: a prospective validation. Blood 2001; 97: 1604-10.

12.- Caillot D, Couaillier J F, Bernard A, Casasnovas O, Denning DW, Mannone L, et al. Increasing volume and changing characteristics of invasive pulmonary aspergillosis on sequential thoracic computed tomography scans in patients with neutropenia. J Clin Oncol 2001; 19: 253-9.

13.- Nucci M. Emerging moulds: Fusarium, Scedosporium and Zygomycetes in transplant recipients. Curr Opin Infect Dis 2003; 16: 607-12.

14.- Silva V, Díaz M C, Febré N y red de diagnóstico en micología médica. Vigilancia de la resistencia de levaduras a antifúngicos. Rev Chil Infect 2002; 19: 149-56.

15.- Rabagliati R, Fuentes G, Guzmán AM, Orellana E, Oporto J, Aedo I, et al. Enfermedad fúngica invasora en pacientes hemato-oncológicos y receptores de trasplante de precursores hematopoyéticos bajo la perspectiva de los criterios diagnósticos EORTC/MSG. Rev Chil Infect 2009; 26: 212-9.

16.- Santolaya M E, Rabagliati R, Bidart T, Paya E, Guzmán A M, Morales R, et al. Consenso Manejo racional del paciente con cáncer, neutropenia y fiebre. Rev Chil Infect 2005; 22: 79-113.

17.- De Pauw B, Walsh T J, Donnelly P, Stevens D A, Edwards J E, Calandra T, et al. Revised definitions of invasive fungal disease from the European Organization for Research and Treatment of Cancer/Invasive Fungal Infections Cooperative Group and the National Institute of Allergy and Infectious Diseases Mycoses Study Group (EORTC/MSG) Consensus Group. Clin Infect Dis 2008; 46: 1813-21.

18.- Borges M. Actualización sobre el tratamiento de las infecciones fúngicas graves. Rev Esp Quimioter 2008; 21: 14-25.

19.- Reboli A C, Rotstein C, Pappas P G, Chapman S W, Kett D H, Kumar D. Anidulafungin Study Group. Anidulafungin versus fluconazole for invasive candidiasis. N Engl J Med 2007; 356: 2472-82.

20.- De hoog G S, Guarro J, Gene J, Figueras M J. Atlas of clinical fungi: Centralbureau voor Schilmmelcultures/Universitat Rovira y Virgili. Baarn and Delf, The Netherlands, Universitat Rovira y Virgili, Reus, Spain, 2000.

21.- Piontelli E. Aportes morfotaxonómicos en el género Aspergillus Link: claves para las especies ambientales y clínicas más comunes. Bol Micol 2008; 23: 49-66.

22.- Gadea I, Cuenca-Estrella M, Martín E, Pemán J, Pontón J, Rodríguez-Tudela J L. Procedimientos de diagnóstico microbiológico de las micosis y estudios de sensibilidad a los antifúngicos. Enferm Infec Microbiol Clin 2007; 25: 336-40.

23.- National Committee for Clinical Laboratory Standards. Methods for antifungal disk diffusion susceptibility testing of yeasts: Approved standard M44-A. NCCLS, Wayne, PA, USA. 2004.

24.- Tapia C. Actualización en pruebas de susceptibilidad antifúngica. Rev Chil Infect 2009; 26: 144-50.

25.- Arikan S. Current status of antifungal susceptibility testing methods. Med Mycol 2007; 45: 569-87.

26.- Patterson T F, Kirkpatrick W R, White M, Hiemenz J W, Wingard J R, Dupont B, et al. Invasive aspergillosis. Disease spectrum, treatment practices, and outcomes. Medicine (Baltimore) 2000; 79: 250-60.

27.- Cornely O, Maertens J, Winston D, Perfect J, Ullmann A, Walsh T, et al. Posaconazole $v s$ fluconazole or itraconazole prophylaxis in patients with neutropenia. N Engl J Med 2007; 356: 348-59.

28.- Groll A H, Shah P M, Mentzel C, Schneider M, Just-Nuebling G, Huebner K. Trends in the postmortem epidemiology of invasive fungal infections at a university hospital. J Infect 1996; 33: $23-32$

29.- Walsh T, Hiemenz J, Seibel N, Perfect J, Horwith G, Lee L, et al. Amphotericin B lipid complex for invasive fungal infections: analysis of safety and efficacy in 556 cases. Clin Infect Dis 1998; 26: 1383-96.

30.- Cornejo M, López J, Navarro S, García de O D, Patiño P. Caracterización clínico-molecular de la enfermedad granulomatosa crónica autosómica recesiva causada por déficit de p47-phox. Rev Med Chile 2000; 128: 490-8.

31.- Pérez A, Contreras A M, Zolezzi R P, Cruz C, Fierro C, Faúndez C, et al. Infección por hongos en catéteres venosos centrales. Rev Chil Pediatr 2002; 73: 489-94.

32.- Coyle E. Invasive candidiasis and the utility of antifungal susceptibility testing in the ICU. J Pharm Pract 2010; 23: 33-7.

33.- Tiraboschi I N, Bennett J E, Kauffman C A, Rex J H, Girmenia C, Sobel J D, et al. Deep Candida infections in the neutropenic and nonneutropenic host: an ISHAM symposium. Med Mycol 2000; 38: 199-204.

34.- Wheat J. Endemic mycosis in AIDS: a clinical review. Clin Microbiol Rev 1995; 8: 146-59.

35.- González A, Tobón A M. Infecciones micóticas oportunistas en pacientes con VIH/SIDA. Infect 2006; 10: 279-87.

36.- Cruz R, Opazo H, Barthel E, Campos S, Piontelli E. Reporte clínico: histoplasmosis diseminada alóctona en un paciente con síndrome de inmunodeficiencia adquirida. Bol Micol 2006; 21: 77-84.

37.- Pinel C, Fricker-Hidalgo H, Lebeau B, Garban F, Hamidfar R, Ambroise-Thomas P, et al. Detection of circulating Aspergillus fumigatus galactomannan: value and limits of the Platelia test for diagnosing invasive aspergillosis. J Clin Microbiol 2003; 41: 2184-6.

38.- Ansorg R, van der Boom R, Rath P M. Detection of Aspergillus galactomannan antigen in foods and antibiotics. Mycoses 1997; 40: 353-7.

39.- Herbrecht R, Denning D W, Patterson T F, Bennett J E, Greene R E, Oestmann J-W, et al. Voriconazole versus amphotericin B for primary therapy of invasive aspergillosis. N Engl J Med 2002; 347: 408-15

40.- Walsh T J, Anaissie E J, Denning D W, Herbrecht R, Kontoyiannis D P, Marr K A, et al. Treatment of aspergillosis: Clinical practice guidelines of the Infectious Diseases Society of America. Clin Infect Dis 2008; 46: $327-60$.

41.- Lass-Flörl C. The changing face of epidemiology of invasive fungal disease in Europe. Mycoses 2009; 52: 197-205.

42.- Tortorano A M, Peman J, Bernhardt H, Klingspor L, Kibbler C C, Faure O, et al. Epidemiology of candidaemia in Europe: 
results of 28-month European Confederation of Medical Mycology (ECMM) hospital-based surveillance study. Eur J Clin Microbiol Infect Dis 2004; 23: 317-22.

43.- Upton A, Kirby K, Carpenter P, Boeckh M, Marr K. Invasive aspergillosis following hematopoietic cell transplantation: outcomes and prognostic factors associated with mortality. Clin Infect Dis 2007; 44: 531-40.

44.- Pappas P G. Invasive candidiasis. Infect Dis Clin North Am 2006; 20(3): 485-506.

45.- Playford E G, Sorrell T C. Optimizing therapy for Candida infections. Semin Respir Crit Care Med 2007; 28: 678-88.

46.- St-Germain G, Laverdiere M, Pelletier R, Bourgault A M, Libman M, Lemieux C, et al.
Prevalence and antifungal susceptibility of 442 Candida isolates from blood and other normally sterile sites: Results of a 2 year (1996 to 1998) multicenter surveillance study in Quebec, Canada. J Clin Microbiol 2001; 39: 949-53.

47.- Gómez J, García-Vázquez E, Espinosa C, Ruiz J, Canteras M, Hernández-Torres A, et al. Nosocomial candidemia at a general hospital: The change of epidemiological and clinical characteristics. A comparative study of 2 cohorts (1993-1998 versus 2002-2005). Rev Iberoam Micol 2009; 26: 184-8.

48.- Aroca T S, Piontelli L E, Cruz Ch R. Case report: Trichoderma longibrachiatum infection in a paediatric patient with peritoneal dialysis. Bol Micol 2004; 19: 13-7.
49.- Tanis B C, Van der pijl H, Van Ogtrop M L, Kibbelaar R E, Chang P C. Fatal fungal peritonitis by Trichoderma longibrachiatum complicating peritoneal dialysis. Nephrol Dial Transplant 1995; 10: 114-6.

50.- Abril V, Ortega E, Segarra P, Pedro F, Sabater V, Herrera A. Rhinocerebral mucormycosis in a patient with AIDS: A complication of diabetic ketoacidosis following pentamidine therapy. Clin Infect Dis 1996; 23: 845-6.

51.- González C, Rinaldi M, Sugar A. Zygomycosis. Infect Dis Clin North Am 2002; 16: 895-914.

52.- Bravo M, Ferrer S, Etchard M, Trujillo S. Mucormicosis rinocerebral. Comunicación de 4 casos. Rev Méd Chile 1999; 127: 712-8. 